\title{
The integration of UAVs to the C-ITS Stack
}

\author{
Felipe Valle*, Martin Cooney*, Konstantin Mikhaylov ${ }^{\dagger}$, and Alexey Vinel ${ }^{*}$ \\ * School of Information Technology, Halmstad University, Sweden \\ ${ }^{\dagger}$ Centre for Wireless Communications, University of Oulu, Finland
}

\begin{abstract}
In this paper, we conceptualise and propose integrating UAVs with Intelligent Transportation Systems (ITS) based on using the Cooperative-ITS (C-ITS) framework. We start by discussing the state of the art and pinpointing some of the reasons for integration and the applications that the envisaged integration would enable. Next, we recall the critical aspects of the state of the art C-ITS connectivity and discuss how seamless integration of UAVs into C-ITS can be achieved. Notably, we show that encapsulation of UAVs in C-ITS does not imply significant changes for the currently existing mechanisms and data formats. Finally, we discuss some of the open research challenges related to the integration and operation of the integrated systems and pinpoint some mechanisms which can help to address these.
\end{abstract}

Index Terms-Drones, UAV, ITS, C-ITS, integration, V2X, CAM, robots, mobile, wireless

\section{INTRODUCTION}

The increasing of safety and efficiency and enabling autonomous mobility over ground, air and water has been a significant trend over the recent years. Some new solutions have been proposed to tackle this challenge, especially in Intelligent Transportation Systems (ITS). An illustrative example of this are vehicular networks (VNs) designed to enable information exchange via Vehicle-to-Vehicle (V2V), and Vehicle-to-Infrastructure (V2I) communications [1], which are about to leave laboratories and start commercial roll-outs.

Currently, one of the most mature ITS development architectures is the ETSI C-ITS (Cooperative ITS) framework [2]. C-ITS is, at its core, a collection of technologies and standards that regulate the information exchange between road users and infrastructure. Its main goal is to facilitate the cooperation between any two or more ITS sub-systems (personal, vehicle, roadside, etc.) based on a single unified common platform and a set of notations and vocabulary.

Meanwhile, another domain that has experienced substantial growth in the past years is robotics in general and Unmanned Aerial Vehicles (UAVs) or drones. Starting as primarily military equipment in the early 2000s, drones are widely employed for versatile services in both piloted and autonomous modes nowadays. However, their safety and reliability are today still limited due to the lack of the means allowing them to detect each other, as well as the limited localization and communication capabilities they feature [3].

In this paper, we approach this challenge through conceptualizing and investigating the prospects of applying the CITS mechanisms to drones with the ultimate goal to enable the seamless integration between the ITS and the drones. The contribution of the current paper is, thus, threefold:

978-1-6654-4131-5/21/\$31.00 @2021 IEEE
- We conceptualize the integration between UAVs and ITS based on C-ITS.

- We discuss two alternative ways how drones can be integrated into ITS in the context of C-ITS, and pinpoint respective pros and cons. Notably, we show that encapsulation of drones in C-ITS does not imply major changes for the currently existing mechanisms and data formats.

- We identify several use cases and novel applications enabled through UAVs and ITS integration and pinpoint key research challenges (and some prospective directions to address these) for the future.

The remainder of this paper is organized as follows. In Section II, we review related works. In Section III, we pinpoint several illustrative application scenarios enabled by integrating UAVs and ITS and C-ITS stack. Section IV discusses alternative ways to handle the integration and assesses the need for new mechanisms to allow this. Section V discusses open research challenges for the future, while Section VI concludes this paper with a summary of key takeaways.

\section{RELATED WORKS}

Drones are considered to be an essential element of Internetof-Things (IoT). They are suitable for undertaking various vital services, some of which are relevant also for ITS, such as low altitude surveillance and post-disaster rescue when equipped with dedicated sensors and communication devices [4]. Moreover, both analytical and field studies have validated the capabilities of drones to support broadband wireless communications or form an own network - the so-called Flying Ad-Hoc Networks (FANETs) [5].

Earlier studies have already suggested using drones in VNs to improve V2V connectivity, infrastructure coverage, network information collection ability, and interworking efficiency. All these are enabled since drones exhibit such advantageous characteristics as: dynamic deployment according to real-time requirements, as well as the ability to easily establish lineof-sight (LOS) links [6] and to quickly form ad-hoc networks within drone swarms [7].

Because of these valuable features, drone communication is considered an attractive candidate for boosting VN performance metrics and safety application performance. In fact, in the state of the art literature, we can identify three typical ITS use-case classes that take advantage of UAV deployments:

1) Alert Broadcasting: Drones can be used to monitor traffic conditions through cameras or sensors. When a drone detects (or is notified about) an emergency, it can directly broadcast safety alerts over LOS links to all 
vehicles (and other drones) within its coverage range, ensuring safety message dissemination over large areas at a higher reliability and throughput [8], [9].

2) Transportation Surveillance: Hovering drones can collect real-time traffic, environment and network information. Algorithms and techniques can be designed to use this information to optimize network performance and improve traffic flow [10], [11].

3) Provisioning of Connectivity Services: Dynamic deployment of a drone or a drone swarm can provide mobile infrastructure capable of mitigating the impact produced by the dynamic topology of VNs while also providing support in regions where the infrastructure is lacking or is temporarily unavailable/overloaded [6], [12], [13].

For instance, on the topic of message broadcasting, Zhang et al. designed an enhanced UAV-aided data dissemination protocol by employing UAVs as flying relays with data caching capability in VANETs [8]. Meanwhile, Zhou et al. studied the use of drones for emergency information dissemination in multihop multicast networks, using Monte Carlo simulations to validate the accuracy of their proposed framework [9].

Substantial research has also been conducted on using UAVs to monitor traffic. For example, Oubbati et al. [10] investigate the use of UAVs to detect and assist accidents on the road by introducing a routing scheme that ensures a high level of communication stability accounting for both the high mobility and the restricted energy capacity of UAVs. Meanwhile, Bashir et al. developed an energy-efficient collaborative scheme between UAVs and VANETs for dissemination of real-time surveillance data using unicast messaging from base stations (BS) to avoid flooding the network [11].

More recently, the focus has turned towards developing hybrid architectures to support the existing VN infrastructure. Saputro et al. designed a secure hybrid communication infrastructure for mobile road-side units (RSUs) that are based on drones [12]. The architecture tackles interoperability issues and coordination between vehicular communications, wireless mesh and LTE. Meanwhile, Weisen et al. introduced the Drone Assisted Vehicular Network (DAVN) [13]. This hybrid architecture provides ubiquitous connections for vehicles by efficiently integrating the communication and networking technologies of drones and connected vehicles. Lin et al. [14] proposed a collaborative communication scheme for multiple drones to assist VANETs, modelling the problem as a multimodal optimization problem. To solve it, they developed a swarm-based optimization algorithm named Multimodal Nomad Algorithm (MNA). Meanwhile, Rui and colleagues [15] designed an advanced UAV-aided cooperative data dissemination scheduling strategy to improve the data dissemination performance in VANETs using spatial dynamic programming for the trajectory scheduling of UAVs.

However, none of these hybrid architectures provides integration between UAVs and VNs - the UAVs are still seen mainly as an extension of the VNs. Therefore, we consider that a design of efficient interworking mechanisms benefiting both parties - the drones and the ITS - is especially bene-

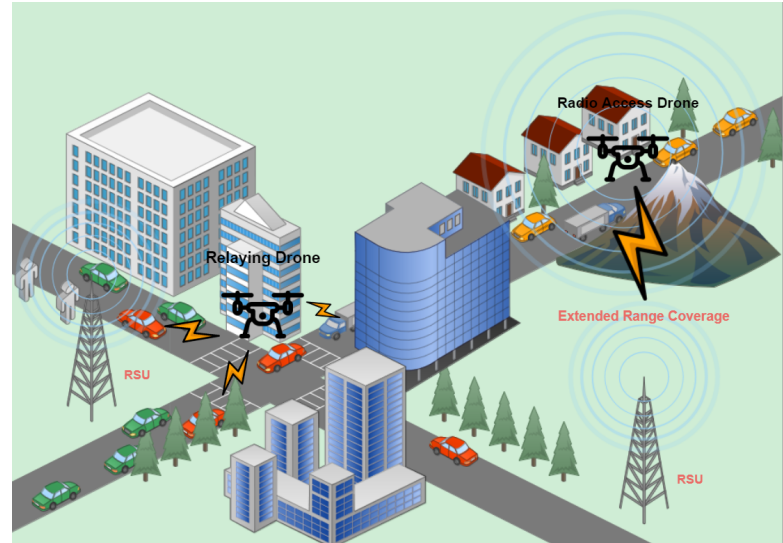

Fig. 1: Illustrative Drone Assisted VN Scenario

ficial. Notably, this will allow drones to also benefit from being integrated into vehicular communications. The use of cooperative awareness applications and services can improve drone mobility safety by expanding the system's access to realtime location and sensing information available to an ITS.

\section{C-ITS-DRONE INTEGRATION USE CASES}

It is easy to observe the advantage of incorporating drones to assist VNs, especially in cooperative awareness scenarios where the introduction of LOS links can significantly boost reliability and reduce latency. Fig.1 shows a typical example of a UAV-assisted VN deployment.

As we saw in the previous section, current research in the area has mainly focused on developing schemes/algorithms to address specific applications instead of analyzing the possibility of having all of the network elements integrated within a single unified framework. In all of these use case scenarios, the drones access and interact with the $\mathrm{VN}$ users and resources through various links. Thus, the interworking problem persists: We need efficient interworking mechanisms between drones and VNs to fulfil the strict requirements of vehicular services and applications on the one hand and enable efficient and safe operation of drones on the other hand.

Nevertheless, if drones were to be integrated directly into the C-ITS stack, this Interworking between Drones and Vehicular Networks challenge would be automatically solved because (i) UAVs would have direct access to the same network resources as regular vehicle users, eliminating potential interoperability problems, and (ii) this equips drones with proved-to-work mechanisms to discover each other and other elements and actors in their vicinity, thus supporting safety. Seamless integration also gives drones access to the same message dictionary developed by ETSI for C-ITS stations, which erases the need to design different messaging systems for new applications. Importantly, this approach also enables to avoid deployment of another connectivity infrastructure to be used by drones, deployment and support of which would have added to the carbon dioxide emission bill.

UAVs would also positively impact some state of the art metrics used for VANETs, such as, age of information (AOI) 
[16] which measures the timeliness of the received information. Using AOI as a performance metric in VANETs [17], [18] has proven effective in meeting the strict C-ITS application requirements. UAV integration would boost $\mathrm{AOI}$ by ensuring the availability of LOS links. Thus, decreasing the probability of receiving outdated information and subsequently decreasing the uncertainty of a vehicle's exact location and velocity.

Notably, a seamless integration of UAVs and VNs also enables several new application UAV-assisted use cases that otherwise would not be possible due to added latency and the lack of interworking mechanisms.

1) Increased UAV Safety Operation: Seamless integration and access to the C-ITS services and resources would improve the safety of drone operations. Drones will be able to use any type of ETSI message, including Decentralized Environmental Notifications (DEN), Signal Request and Response (SSM \& SRM), Map Data (MAP), etc. Thus, UAVs can take advantage (in both D2D and D2V) of the safety services designed for vehicles, such as cooperative awareness applications and hazard warning notifications. Even though vehicle sensor information is at the ground level, information about obstacles/hazards on the infrastructure could still be used by UAVs when coordinating navigation paths, specially, if we use a combination of "static" drones that rise/return from a launching pad working as BS (with different altitude values) with more "dynamic" drones that move based on the traffic situation to optimize communication, as well as drones doing other tasks, like monitoring a city traffic situation or carrying packages.

2) UAV-assisted Emergency Vehicle Warning: Some work has already been done on the use of UAVs to detect road accidents and plot the fastest intervention paths for emergency response teams [10]. However, if a UAV can send the same messages that regular vehicle users use, then many novel opportunities open up. For example, UAVs could act as "mobile sirens" equivalent, leading emergency vehicles (EV) while broadcasting EV warnings and clearing the roadin advance. It could be used to talk directly to the traffic light controller to request signal priority for EVs with more anticipation or even set up roadblocks by signaling that roads are closed in order to prevent traffic jams or more accidents.

3) Collective Perception Applications: Collective perception is based on the idea of sharing sensor-detected objects via V2X communications [19]. In this scenario, drones serve two primary purposes: one, increasing the number of detected objects due to their privileged positioning and second, boosting the reliability of the shared data via LOS links [20].

4) Support of UAV operations by ITS infrastructure: Notably, the drones can not only provide services but also get support from ITS. To give an example, talking the same "language", a UAV can opportunistically land on a vehicle (e.g., a bus or a taxi) and negotiate trans-

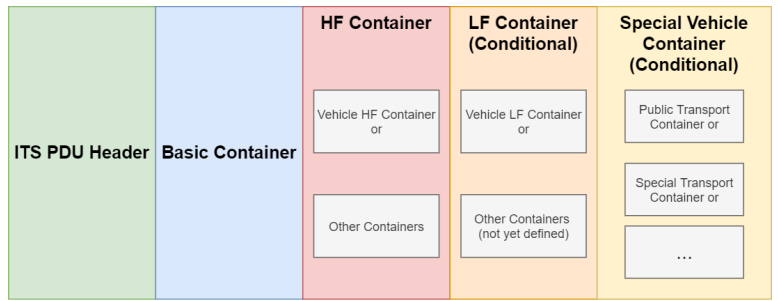

Fig. 2: Data Structure of a Cooperative Awareness Message

portation to a new location. The use of a vehicular charging station by a drone represents another illustrative example. Thus, in the future there will also be a need for more sophisticated and updated UAV operation mechanisms/algorithms in order to support these more advance specifications.

It is worth noting that we can distinguish three drone deployment scenarios in a UAV-Assisted VN Architecture. The first one is Drone Assisted V2V Networking, where drones essentially work as network relays, maintaining LOS links between vehicle users. The second one is Drone Assisted V2I Networking, in which vehicle users communicate with drones as if they were part of the ITS infrastructure; in this scenario, drones essentially become mobile base stations (BS) to extend coverage or plug coverage holes as needed. The third deployment scenario is the Drone Swarm Network; in this instance, a VANET is deployed using drone-to-drone links, and drones work both as network access gates providing spectrum resources and as relaying nodes for other users. The last scenario also differs from the first two because, technically, the swarm network could also operate on a different spectrum from IEEE $802.11 \mathrm{p}$ and cellular bands used for C-ITS. This work focuses on the two former scenarios, representing the first step towards integrating drones and C-ITS and creating a basis for further swarm networking implementation. In the following section, we investigate and discuss the possibility of drones being successfully and seamlessly integrated into the ETSI C-ITS framework, analysing the key architectural requirements and providing potential solutions to some of the technical challenges that it brings.

\section{C-ITS AND DRONES INTEGRATION}

\section{A. C-ITS Basics}

ITS embrace a wide variety of communications-related applications intended to increase travel safety, improve traffic efficiency and maximize the benefits to all types of users. The main objective of this paper is to highlight how the potential integration of drones into the ETSI C-ITS framework [2] can be carried out and identify the key challenges, open research questions and possible solutions.

In C-ITS the vehicles' onboard units (OBUs) communicate directly with each other and/or with the infrastructure, i.e., the Road Side Units (RSUs). The backbone of the C-ITS framework is the so-called cooperative awareness message (CAM) [21]. CAMs are messages exchanged in the ITS network 
between ITS stations (ITS-S) to create and maintain awareness of each other and support vehicles' cooperative performance using the road network. A CAM contains information about the status and attributes of the originating stations and provide some insight into the intentions of the different actors. Thus, any type of drone integration will imply ability to send and receive the CAM structures shown in Fig. 2.

It can be seen that a CAM is composed of one common ITS Protocol Data Unit (PDU) header and multiple containers. The ITS PDU header is a common header that includes the protocol version, the message type and the identifiers (IDs) of the originating ITS-S. Depending on the type of station (vehicle or infrastructure), the CAM will contain a mandatory basic container and optional high/low-frequency containers. Each container comprises a sequence of optional or compulsory data elements (DE) and/or data frames (DF) [21], [22].

The basic container provides basic information of the originating ITS-S: the type of the originating ITS-S as well as its latest geographical position. Infrastructure ITS-S, i.e., the RSUs, use only this container. Meanwhile, CAMs generated by an OBU include at least a high-frequency vehicle (Vehicle $H F$ ) container, which has all fast-changing (dynamic) status information of the vehicle ITS-S such as heading or speed. The optional low-frequency vehicle (Vehicle LF) container keeps a record of the static or slow-changing data like the status of the exterior lights.

Given that drones are highly dynamic, the first instinct is to disregard the need for LF containers containing mainly static information about the originating ITS. However, its use may be required if there is a need to designate drones for some specialized purposes (e.g., emergency vehicle, safety car, etc.).

\section{B. Suitability of C-ITS Protocol for Drones}

Next, the first open question relates to which role drones will assume within the vehicle infrastructure. Do we consider drones as vehicles within the network, e.g., using them as relaying nodes $(R N)$ able to connect multiple vehicles with LOS links simultaneously; or do we consider them part of the network infrastructure, e.g., as remote radio access nodes (RRAN) that plug coverage holes and enhance V2I connectivity [13]. The pros and cons of these two approaches are discussed in the two following subsections.

1) Drones as Vehicles: Treating drones as flying vehicles implies drones generating the same CAMs as regular vehicle users. However, care must be taken to prevent the network from flooding with these beacon messages. This can be done by reducing the C-ITS beaconing frequency for UAVs in the network, which can be done since (i) unlike the vehicles, the drones more often benefit from LOS conditions and associated communicating range, (ii) the speed of the drones (especially at low altitudes) is usually lower than that of the vehicles. However, we suggest that instead of a fixed beacon period, adaptive beaconing techniques, taking into account the mobility of the drone and the environment around it, would be more beneficial [23].
2) Drones as Infrastructure: The alternative approach implies drones taking the role of RSUs. The RSUs are expected to be stationary and thus, CAMs they broadcast encapsulate only the basic container. Therefore, the RSU role might be reasonable primarily for static drones - the landed or hovering ones. An addition of a high-frequency container, which carries the relevant information about mobility and is needed for a moving UAV, will make the drone look like a vehicle, as discussed in the previous subsection.

Based on the discussion above and for the sake of uniformity, we suggest that a CAM message of a UAV, while in the air or about to take off, should be composed of one basic container and one high-frequency container. This is beneficial because and allows a drone to take either of the roles - a vehicle or an infrastructure element - and even switch between them dynamically in response to the changes in the environment and services a drone will be providing.

The main issue in both scenarios is introducing the altitude element, which is needed when we consider UAVs in the air. Due to obvious reasons, the conventional C-ITS networks imply mobility of the devices in 2D space and thus do not have a ready-made solutions for this, to tackle this challenge, we suggest making use of the facilities layer [24] which provides data collection support to the different C-ITS services including the Cooperative Awareness basic service (CA basic service). The common data dictionary [22], which is a repository of data elements used by the CA basic service, also includes an altitude data frame that contains its value on the WGS84 coordinate system and an accuracy measure.

Lastly, in order to manage spectrum availability, specifically in densely crowded environments, we propose decreasing the transmission power of the UAVs' radio in order to limit their communication range. This has several advantages: (i) it allows us to focus on more bounded service areas which improves the reliability and performance of the safety applications, (ii) it avoids potential handover issues when the drones are in overlapping ranges of infrastructure BS coverage, (iii) it limits the possibility of exhausting network resources due to message flooding and collision. This way we can help ensure that the integration of UAVs won't compromise the safety of vehicle users and pedestrians.

\section{Message Delay Models}

Consider a typical safety application in which every vehicle continuously sends beacon messages to all its neighbors. The most critical requirement is that end-to-end delay for a transmission must not exceed 100ms, otherwise the receiver does not have time to react, especially in case of emergencies. We can also assume that by using UAVs the messages only need one-hop at maximum to reach their destination.

In a C-ITS environment that uses 802.11 p ad-hoc communication, we can simplify the model presented in [25] for the Distributed Coordination Function (DCF) delay to obtain the access time in basic mode (i.e., without RTS/CTS). The DCF mechanism can be summarized as follows: if the channel is detected idle for a period of time $\left(T_{D I F S}\right)$, a station can 
transmit immediately. Otherwise, a collision is detected, and the station will defer until the end of transmission while a random backoff interval is selected. Taking that into account, we can use the following models for $T_{\text {Success }}$, the average time the channel is sensed to be busy due to a successful transmission, and $T_{\text {Collision }}$, the average time the channel is sensed busy by each station during a collision:

$$
\begin{aligned}
T_{\text {Success }}= & T_{D I F S}+\frac{H+P}{C_{d}}+\delta+T_{\text {SIFS }}+\frac{A C K}{C_{c}}+\delta, \\
& T_{\text {Collision }}=T_{D I F S}+\frac{H+P}{C_{d}}+\delta
\end{aligned}
$$

where $H$ is the packet header, $P$ is the payload in number of bits, $C_{d}$ is the capacity of the link (in bits per second) for data channel, and $C_{c}$ is the control channel capacity. The header is $H=P H Y_{h d r}+M A C_{h d r}$, and the payload includes the $I P_{h d r}$. While $\delta$ denotes the propagation delay inside the end device, depends on the PHY layer, and accounts for the time required to signal the state of the channel to the MAC layer.

Moreover, $T_{S I F S}$ is the time the receiver waits to send the ACK package back to the transmitter. Because we are only interested in the broadcast scenario, we can ignore the sending of the ACK package. Thus, equation 1 is reduced to:

$$
T_{\text {Success }}=T_{D I F S}+\frac{H+P}{C_{d}}+\delta .
$$

In a broadcast scenario, the average time the channel is sensed to be busy due to a successful transmission is identical to the average time the channel is sensed to be busy during a collision. For a preliminary analysis we are only interested in getting a good approximation. To do this, we can measure the delay of every user in the network and then take the average of the measurements. If we know that a user senses that the channel is busy $N$ times while attempting a transmission, then the average packet delay is given by:

$$
T=N * T_{\text {Collision }}+\sum_{i=1}^{N}\left(\frac{C W_{M i n}-1}{2}\right) * S T+T_{\text {Success }},
$$

where $S T$ is the length of a time slot and $C W_{M i n}$ is the minimum size of the contention window. During the backoff procedure, the backoff time is uniformly chosen in the range $\left[0, C W_{M \text { in }}-1\right]$ interval. For our approximation, we take the value $\frac{C W_{\text {Min }}-1}{2}$ as it represents the average value over the distribution. In the broadcast scenario, the mechanism only has one backoff stage and $C W_{M i n}$ does not increase after each retransmission attempt. Thus, each time the channel is sensed to be busy a user waits, on average, a time equal to $\frac{C W_{M i n}-1}{2}$ before sensing the channel again.

Thus, we can see the average packet delay is directly proportional to the number of packet collisions encountered when attempting transmission. Furthermore, in an scenario with enhanced availability of LOS links, such as, one where UAVs are deployed into the C-ITS networks. The frequency of the beacons can be adjusted and reduced significantly because individual vehicles are easier to reach, thereby reducing the risk of flooding the network (LOS links enhance communication reliability and thus increase the packet delivery ratio (PDR) of the system). This results in a reduction on the average number of packet collisions i.e. there is less risk of sensing the channel busy if less messages are being sent. Therefore, in the UAV scenario the active presence of LOS links could help decrease the average packet delay (i.e. latency) which is the key requirement for safety applications.

\section{Spectrum Interference \& Allocation}

Since we assume that vehicles and UAVs will be operating using the same communication stack, an obvious issue will be spectrum allocation. We discussed in a previous section that the most direct solution to manage spectrum availability is to control the transmission power of UAVs. However, we expect to be able to use UAVs as C-ITS Infrastructure stations. Thus, UAVs must be able to increase spectrum availability by providing resources themselves (individually or as a swarm). This opens up challenges on resource allocation between users and services. And on how statically or dynamically should this be handled. A potential approach is using a network virtualization point-of-view, UAVs acting as RRANs can be considered by vehicles as abstract access points for resources. Then, it is possible to allocate different virtual network slices to a single drone for each service it provides and dynamically change them based on the environment and situation.

Finally, another important advantage from using UAVs is helping alleviate some of the most important issues regarding the signal degradation associated with multipath propagation. LOS links help reduce shadowing and signal scattering levels which improves the signal quality by decreasing the effects of time dispersion. Thus, improving the quality of the communications and the efficiency of spectrum resource utilization.

\section{Challenges And Open Research Questions}

In what follows, we pinpoint key challenges and open research questions that still need to be addressed to to enable seamless integration of drones into VNs.

Efficient transceiver design: The conventional C-ITS transceivers have been designed to be carried by cars or integrated with road infrastructure. Neither of these use cases requires minimization of the wight, linear dimensions and energy consumption of a C-ITS transceiver. This is clearly no the case with drones, for which each extra gram of wight reduces its operation time. Another major design challenge is the development of an antenna that will be omnidirectional in 3D. However, this challenge can also be addressed by equipping a drone with several antennas.

Authentication and Security: For interoperability reasons, a uniform format for data structures representing security headers and public key certificates needs to be provided as well as enrollment credentials that will need to be coordinated with a centralized certificate authority (CA). As part of the C-ITS infrastructure, drones will become ITS-S themselves; thus, UAVs will need to comply with the security policies 
specified by the ETSI standards [26] to access communication and services.

Establishing Trust and Support of Collaboration: After provisioning a way for drones, vehicles and infrastructure to speak the same language, the next logical step would be to enable collaborative actions between all these. In this way, there arises a need to establish the mechanisms for establishing trust and supporting the collaboration between the different actors. Some novel public key based schemes have already been proposed [27] on the topic. Distributed ledger technologies (DLT) and smart contracts are also promising technologies to tackle this challenge.

\section{CONClusions}

In this work, we conceptualize and propose integrating drones with the ITS based on the use of C-ITS framework. Surprisingly, to the best of our knowledge, this approach has not been proposed yet, In this way, we have first discussed the motivation for this integration by introducing several illustrative applications and use cases, which this can enable. Following this, we have discussed how this integration can be carried out by analyzing two alternative implementation strategies and pinpointing the perspective of C-ITS mechanisms and some relevant changes. Finally, we have identified and discussed some of the further research directions and challenges, which need to be addressed.

Even the preliminary analysis in this paper demonstrates that drones and an ITS can be merged, which would deliver mutual benefit. Specifically, the integration with an ITS would (i) equip the drones with efficient and proven safety mechanisms, (ii) build the basis for more efficient mission execution. The ITS, in its turn, can use some of the services delivered by drones. However, even though we have demonstrated the potential feasibility of this approach in this paper, much work is still needed to address the identified challenges and enable the conceptualized integrated system to be deployed in-field. We hope that this work will attract attention to this problem and motivate further studies in this field.

\section{ACKNOWLEDGEMENT}

The work of K. Mikhaylov has been supported by the Academy of Finland via: (a) 6G Flagship (n.318927); (b) RoboMesh (n.336060); and (c) MRAT-SafeDrone (n.341111) projects. Halmstad University has received funding from the Knowledge Foundation (KKS) in "Safety of Connected Intelligent Vehicles in Smart Cities - SafeSmart" project (201923), Swedish Innovation Agency (VINNOVA) in "Emergency Vehicle Traffic Light Pre-emption in Cities - EPIC" project (2020-22) and the ELLIIT Strategic Research Network.

\section{REFERENCES}

[1] K. Abboud, H. A. Omar, and W. Zhuang, "Interworking of DSRC and Cellular Network Technologies for V2X Communications: A Survey," IEEE Trans. Vehic. Techol., vol. 65, no. 12, pp. 9457-9470, 2016.

[2] "ETSI TR 101607 Intelligent Transport Systems (ITS); Cooperative ITS (C-ITS); Release 1 ," ETSI, Sophia Antipolis Cedex, France, 2020.

[3] E. Vinogradov, F. Minucci, and S. Pollin, "Wireless Communication for Safe UAVs: From Long-Range Deconfliction to Short-Range Collision Avoidance," IEEE Veh. Technol. Mag., vol. 15, no. 2, pp. 88-95, 2020.
[4] N. Motlagh, T. Taleb, and O. Arouk, "Low-Altitude Unmanned Aerial Vehicles-Based Internet of Things Services: Comprehensive Survey and Future Perspectives," IEEE IoT J., vol. 3, no. 6, pp. 899-922, 2016.

[5] L. Gupta, R. Jain, and G. Vaszkun, "Survey of Important Issues in UAV Communication Networks," IEEE Commun. Surveys\&Tutorials, vol. 18, no. 2, pp. 1123-1152, 2016.

[6] I. Bor-Yaliniz and H. Yanikomeroglu, "The New Frontier in RAN Heterogeneity: Multi-Tier Drone-Cells," IEEE Commun. Mag., vol. 54, no. 11, pp. 48-55, 2016.

[7] O. Shrit, S. Martin, K. Alagha, and G. Pujolle, "A new approach to realize drone swarm using ad-hoc network," in Proc. 16th Annu. Mediterranean Ad Hoc Networ. Workshop, 2017, pp. 1-5.

[8] R. Zhang, F. Zeng, X. Cheng, and L. Yang, "UAV-Aided Data Dissemination Protocol with Dynamic Trajectory Scheduling in VANETs," in Proc. Int. Conf. Commun., 2019, pp. 1-6.

[9] X. Zhou, S. Durrani, and J. Guo, "Drone-Initiated D2D-Aided Multihop Multicast Networks for Emergency Information Dissemination," IEEE Access, vol. 8, pp. 3566-3578, 2020.

[10] O. S. Oubbati, A. Lakas, P. Lorenz, M. Atiquzzaman, and A. Jamalipour, "Leveraging communicating uavs for emergency vehicle guidance in urban areas," IEEE Trans. Emerging Topics in Comput., vol. 9, no. 2, pp. 1070-1082, 2021.

[11] N. Bashir and S. Boudjit, "An Energy-Efficient Collaborative Scheme for UAVs and VANETs for Dissemination of Real-Time Surveillance Data on Highways," in Proc. 17th Аnпи. Consumer Commun. Netw. Conf., 2020, pp. 1-6.

[12] N. Saputro, K. Akkaya, R. Algin, and S. Uluagac, "Drone-Assisted Multi-Purpose Roadside Units for Intelligent Transportation Systems," in Proc. 88th Vehicular Techn. Conf., 2018, pp. 1-5.

[13] W. Shi, H. Zhou, J. Li, W. Xu, N. Zhang, and X. Shen, "Drone Assisted Vehicular Networks: Architecture, Challenges and Opportunities," IEEE Network, vol. 32, no. 3, pp. 130-137, 2018.

[14] N. Lin, L. Fu, L. Zhao, G. Min, A. Al-Dubai, and H. Gacanin, "A Novel Multimodal Collaborative Drone-Assisted VANET Networking Model," IEEE Trans. Wireless Commun., vol. 19, no. 7, pp. 4919-4933, 2020.

[15] R. Lu, R. Zhang, X. Cheng, and L. Yang, "Relay in the Sky: A UAV-Aided Cooperative Data Dissemination Scheduling Strategy in VANETs," in Proc. IEEE Int. Conf. Commun., 2019, pp. 1-6.

[16] A. Kosta, N. Pappas, and V. Angelakis, Age of Information: A New Concept, Metric, and Tool, 2017.

[17] N. Lyamin, B. Bellalta, and A. Vinel, "Age-of-information-aware decentralized congestion control in vanets," IEEE Netw. Let., vol. 2, no. 1, pp. $33-37,2020$.

[18] I. Turcanu, A. Baiocchi, N. Lyamin, and A. Vinel, "An age-ofinformation perspective on decentralized congestion control in vehicular networks," in Proc. 19th Mediterranean Commun. Comp. Netw. Conf., 2021, pp. 1-8.

[19] H.-J. Günther, R. Riebl, L. Wolf, and C. Facchi, "Collective perception and decentralized congestion control in vehicular ad-hoc networks," in Proc. IEEE Vehicular Netw. Conf., 2016, pp. 1-8.

[20] F. A. Schiegg, I. Llatser, D. Bischoff, and G. Volk, "Collective Perception: A Safety Perspective," Sensors, vol. 21, no. 1, 2021.

[21] "ETSI EN 302 637-2 Intelligent Transport Systems (ITS); Vehicular Communications; Basic Set of Applications; Part 2: Specification of Cooperative Awareness Basic Service," ETSI, Sophia Antipolis Cedex, France, 2019.

[22] "ETSI EN 102 894-2 Intelligent Transport Systems (ITS); Users and applications requirements; Part 2: Applications and facilities layer common data dictionary," ETSI, Sophia Antipolis Cedex, France, 2018.

[23] M. Sepulcre, J. Gozalvez, O. Altintas, and H. Kremo, "Adaptive beaconing for congestion and awareness control in vehicular networks," in Proc. IEEE Vehicular Netw. Conf., 2014, pp. 81-88.

[24] "ETSI EN 302665 Intelligent Transport Systems (ITS); Communications Architecture ," ETSI, Sophia Antipolis Cedex, France, 2010.

[25] A. M. Abbas and K. A. M. A. Soufy, "Analysis of IEEE 802.11 DCF for ad hoc networks: Saturation," in Proc. IEEE Int. Conf. Internet Multimedia Serv. Architec. Appl., dec 2011, pp. 1-6.

[26] "ETSI TS 103 097 V1.4.1 Intelligent Transport Systems (ITS); Security; Security header and certificate formats," ETSI, Sophia Antipolis Cedex, France, 2020.

[27] C. F. E. de Melo, T. Dapper e Silva, F. Boeira, J. M. Stocchero, A. Vinel, M. Asplund, and E. P. de Freitas, "Uavouch: A secure identity and location validation scheme for uav-networks," IEEE Access, vol. 9, pp. 82 930-82 946, 2021. 